\title{
Commentaries
}

\section{Transabdominal bowel sonography in Crohn's disease}

The general availability of high resolution ultrasound has improved the diagnostic potential of ultrasound in the assessment of bowel disease. Uncertainty persists however as to its limitations and strengths, and its place in relation to other imaging techniques. Barium studies and endoscopy are currently the most reliable techniques for evaluation of mucosal disease but provide limited information about the extent of transmural and peri-intestinal abnormality.

The paper by Gasche et al (see page 112) highlights the accuracy of transabdominal ultrasound in the detection of complications of Crohn's disease. The authors report the ultrasound findings in a highly selected group of 33 patients with known advanced Crohn's disease who underwent surgery and provide detailed correlation between the ultrasound and pathological findings. They report $100 \%$ sensitivity in the diagnosis of strictures and abscesses with specificity of $91 \%$ and $92 \%$, respectively, and an $87 \%$ sensitivity, $90 \%$ specificity in the diagnosis of fistulae. These are impressive results, although the selection bias is recognised by the authors in that patients requiring surgery are likely to have more severe and obviously detectable disease on ultrasound. Patients who had undergone surgery within the previous six months, where the ultrasound findings may be more difficult to interpret, were excluded from the study as were patients with deep pelvic disease where the contribution of transabdominal ultrasound is limited.

In this study, any ultrasound detected hypoechoic periintestinal lesion measuring less than $2 \mathrm{~cm}$ in diameter was considered to be a fistula. Lesions measuring greater than this arbitrary cut off point were diagnosed as abscesses. Pathologically all lesions which penetrated the muscularis propria were considered fistulae and characterised by a central lumen less than $1 \mathrm{~cm}$ in diameter containing necrotic debris with a border of granulation tissue. The authors' justification for this definition is that the transmural extension of disease into the mesentery is the defining event which influences the subsequent development of complications and thus affects management. However, combining blind ending sinus tracks with fistulae in this study makes it more difficult to compare with others which use the more conventional definition of a fistula as a track communicating between two epithelial surfaces. For example, in a prospective study of transabdominal ultrasound, Maconi et al reported fistulae in only $50 \%$ of patients whereas the sensitivity for the detection of strictures (94\%) and abscesses (83\%) was similar. ${ }^{1}$

In the absence of surgery there is no established "gold standard" for the diagnosis of fistulae. It seems likely that barium studies underestimate the extent of transmural disease, suggested in this study by the identification of a fistula on barium examination in only one of five non-operated patients in whom a "fistula" was diagnosed on ultrasound. Nevertheless, the ultrasound findings must be interpreted with caution as they are non-specific and may be indistinguishable from postoperative granulation tissue and other entities such as diverticulitis.

The 180 non-operative patients studied with ultrasound form a separate group not further evaluated in this study. However, they provide some indication of the value of ultrasound in a less selected population, despite the lack of pathological correlation. Ultrasound diagnosed nine stric- tures, all of which were confirmed on subsequent barium studies. In five patients in whom ultrasound was normal, bowel obstruction was identified by other studies within six months of the ultrasound examination. No other significant complication was demonstrated in the 168 patients who had a normal ultrasound study.

The role of ultrasound in defining the presence and extent of disease at primary diagnosis and the relation of the ultrasound appearances to Crohn's disease activity have been the subject of several studies. ${ }^{2-4}$ In a prospective study by Sheridan et al, of ultrasound as the initial investigation of patients with suspected Crohn's disease or recurrence of established disease, ultrasound sensitivity was $78 \%$, rising to $87 \%$ at the end of the study as a result of the learning curve, with a specificity of $91 \% .{ }^{2}$ Other studies report an overall sensitivity and specificity of transabdominal ultrasound in the diagnosis of inflammatory bowel disease of between $90.3 \%$ and $95 \%$ and $90.5-93 \%$ if assessment of rectal disease is excluded. Ninety five per cent of abscesses were detected, although deep pelvic collections were obscured. ${ }^{3}{ }^{4}$

Attempts to differentiate between ulcerative colitis and Crohn's disease on the basis of the echomorphology of the bowel wall have met with variable success. ${ }^{3-6}$ Preservation or loss of the echostratification of the bowel wall bears some relation to the depth and severity of the inflammatory process but cannot differentiate accurately ulcerative colitis, Crohn's disease or other inflammatory disorders. ${ }^{45}$

In assessment of disease activity, loss of stratification and the extent of wall thickness correlates with the severity of inflammatory change ${ }^{5}$ although the correlation with disease activity is relatively weak. ${ }^{7}$ In clinically quiescent disease, notably increased bowel wall thickness suggests the presence of fibrosis which is likely to be unresponsive to medical therapy. ${ }^{7}$

Hypervascularity of the bowel wall based on visual evaluation of colour Doppler flow imaging seems to parallel inflammatory disease activity but is difficult to quantify. ${ }^{3}$ Van Oostayen et al have suggested that Crohn's disease activity can be objectively determined by assessment of superior mesenteric artery flow with Doppler ultrasound reflecting the increase in mesenteric blood flow recognised in the acute stage of disease. ${ }^{8}$ A longitudinal study of the rate of increased superior mesenteric artery flow following food challenge demonstrated prolongation of the time to peak flow with disease remission, although the test is technically demanding and time consuming. ${ }^{9}$

The lack of radiation and ready availability makes ultrasound an attractive technique, particularly in a disease which affects young patients of reproductive age who may require serial studies. The technique is highly operator dependent and in both primary diagnosis and the detection of complications a high index of suspicion together with detailed study and time commitment are required to achieve accuracy rates comparable with published data. Ultrasound compliments information available from barium studies and may substitute in patients for whom contrast radiology is contraindicated. The accuracy of transabdominal ultrasound in the early detection of intra-abdominal complications justifies its more frequent use although the surgeon may require confirmation of the ultrasound findings by computed tomography scanning before planning definitive management.

Department of Diagnostic Imaging,

A McLEAN

St Bartholomew's Hospital,

Queen Elizabeth Wing,

West Smithfield,

London EC1A $7 B E$, UK 
1 Maconi G, Bollani S, Bianchi Porro G. Ultrasonographic detection of intestinal complications in Crohn's disease. Dig Dis Sci 1996;41:1643-8.

2 Sheridan MB, Nicholson DA, Martin DF. Transabdominal ultrasonography as the primary investigation in patients with suspected Crohn's disease or recurrence: a prospective study. Clin Radiol 1993;48:402-4.

3 Schwerk WB, Beckh K, Raith M. A prospective evaluation of high resolution sonography in the diagnosis of inflammatory bowel disease. Eur $\mathcal{F}$ Gastroenterol Hepatol 1992;4:173-82.

4 Solvig J, Ekberg O, Lindgren S, et al. Ultrasound examination of the small bowel: comparison with enteroclysis in patients with Crohn's disease. Abdom Imaging 1995;20:323-6.

5 Hata J, Haruma K, Yamanaka H, et al. Ultrasonographic evaluation of the bowel wall in inflammatory bowel disease: comparison of in vivo and in vitro studies. Abdom Imaging 1994;19:395-9.
6 Khaw KT, Yeoman LJ, Saverymuttu SH, et al. Ultrasonic patterns in inflammatory bowel disease. Clin Radiol 1991;43:171-5.

7 Maconi G, Parente F, Bollani S, et al. Abdominal ultrasound in the assessment of extent and activity of Crohn's disease: clinical significance and implication of bowel wall thickening. Am f Gastroenterol 1996;91:1604-9.

8 Van Oostayen JA, Wasser MNJM, van Hogezand RA, et al. Doppler sonography evaluation of superior mesenteric artery flow to assess Crohn's disease activity: correlation with clinical evaluation, Crohn's disease activity index and $\alpha_{1}$-antitrypsin clearance in faeces. AfR Am f Roentgenol 1997; ity index and

168:429-33.
9 Britton I, Maguire C, Adams C, et al. Assessment of the role of reliability of sonographic post-prandial flow response in grading Crohn's disease activity. Clin Radiol 1998;53:599-603.
Last year's Nobel Prize in Physiology or Medicine was awarded to Robert F Furchgott, Louis J Ignarro and Ferid Murad for their discoveries concerning nitric oxide as a signalling molecule in the cardiovascular system. In 1977 it was found that nitric oxide could activate guanylate cyclase, the enzyme responsible for the formation of cyclic guanosine monophosphate (cGMP) from guanosine triphosphate, and it was hypothesised that sodium nitroprusside and nitroglycerin could exert their action on guanylate cyclase by donating nitric oxide. ${ }^{1}$ It was suggested that the accumulation of cGMP in a smooth muscle cell was associated with relaxation of that cell. ${ }^{2}$ In 1980 Furchgott and colleagues ${ }^{3}$ showed that acetylcholine dilatated blood vessels only if the endothelium was intact. They hypothesised that the endothelial cells produced a signalling substance (EDRF, endothelial derived relaxing factor) which caused the smooth muscle cells of the vessel wall to relax. Later, it would be established that the EDRF was indeed nitric oxide. ${ }^{45}$

Gaseous nitric oxide may diffuse from one cell to another, an entirely new principle for signal transmission in biological systems. ${ }^{6}$ The substrate for the synthesis of nitric oxide is L-arginine and the formation of nitric oxide and L-citrulline is governed by nitric oxide synthase (NOS). ${ }^{7}$ Derivatives of L-arginine, like $N^{\mathrm{G}}$-nitro-L-arginine (L-NNA), $N^{\mathrm{G}}$-nitro-Larginine methyl ester (L-NAME), and $N^{\mathrm{G}}$-monomethyl-Larginine (L-NMMA), block the enzyme and so inhibit the production of nitric oxide.

Soon it became evident that nitric oxide is involved in the function of many organ systems, not least the gastrointestinal tract. ${ }^{8}$ Like the wall of blood vessels, the intestinal wall is composed of layers of smooth muscle cells. The contractile activity of the gut has an intriguing pattern which is known as the migrating motor complex (MMC). Animal studies have shown that inhibitors of NOS initiate premature phase IIIs of the MMC whereas donors of nitric oxide disrupt the MMC.

In this issue, Russo et al (see page 72) report the results of a study in which they used L-NMMA to inhibit NOS in seven healthy volunteers. The study validates results from animal models used to investigate the role of NO in gut motility and it confirms in humans that $\mathrm{NO}$ is involved in the modulation of MMC. More importantly, their work pioneers studies of gastrointestinal motility using inhibitors of NOS in humans.

Since the discovery of the $\mathrm{MMC}^{9}$ a large number of studies have indicated that virtually any peptide, hormone or transmitter substance in the gut or the brain is involved in the regulation of fasting motility. The purported regulators of the MMC include insulin, acetylcholine, opioids, calcitonin, neurotensin, substance $\mathrm{P}$, motilin, pancreatic polypeptide, growth hormone-releasing factor, somatostatin, secretin, prostaglandins, serotonin, cholecystokinin, corticotropin-releasing factor, peptide YY, norepinephrine, dopamine, neuropeptide Y, epidermal growth factor, bombesin, vasoactive intestinal polypeptide, and NO. In addition, luminal factors like bile and systemic factors like glucose concentrations may influence the pattern of fasting motility. Some of the factors have been shown to act via the enteric nervous system, others via neural connections from the brain. NO seems to act directly on smooth muscle cells and this indicates that $\mathrm{NO}$ may act as a last messenger in a complex chain of events.

However, it is difficult, not to say impossible, at least from the view of a linear analogue control system, to understand the motor function of the gut. The large number of factors and the many levels at which effectors may act suggest that a highly complex organisation underlies the MMC. At the same time the MMC seems to be the most basic programme of the enteric nervous system..$^{10}$ Even a bowel that has become devoid of all nerve supply will exhibit the recurrent MMC pattern. ${ }^{11}$ The MMC is an inherent feature of the gut wall and it is tempting to suggest that the MMC may not be regulated at all, at least not in the sense that something is responsible for its initiation. All that can be done is to modulate the theme that is already there.

G LINDBERG

Department of Medicine,

Division of Gastroenterology and Hepatology,

Karolinska Institute,

Huddinge University Hospital,

SE 14186 Huddinge,

Sweden

1 Katsuki S, Arnold W, Mittal C, et al. Stimulation of guanylate cyclase by sodium nitroprusside, nitroglycerin and nitric oxide in various tissue preparations and comparison to the effects of sodium azide and hydroxylamine. f Cyclic Nucleotide Res 1977;3:23-35.

2 Murad F, Arnold WP, Mittal CK, et al. Properties and regulation of guanylate cyclase and some proposed functions for cyclic GMP. Adv Cyclic Nucleotide Res 1979;11:175-204.

3 Furchgott RF, Zawadzki JV. The obligatory role of endothelial cells in the relaxation of arterial smooth muscle by acetylcholine. Nature 1980;288: 373-6.

4 Ignarro LJ, Buga GM, Wood KS, et al. Endothelium-derived relaxing factor produced and released from artery and vein is nitric oxide. Proc Natl Acad Sci USA 1987;84:9265-9.

5 Furchgott RF. Studies on relaxation of rabbit aorta by sodium nitrite: the basis for the proposal that the acid-activatable inhibitory factor from retractor penis is inorganic nitrite and the endothelium-derived relaxing factor is nitric oxide. In: Vanhoutte PM, ed. Vasodilation: vascular smooth muscle, peptides, and endothelium. New York: Raven Press, 1988:401-14.

6 Ignarro LJ. Nitric oxide. A novel signal transduction mechanism for transcellular communication. Hypertension 1990;16:477-83.

7 Palmer RM, Moncada S. A novel citrulline-forming enzyme implicated in the formation of nitric oxide by vascular endothelial cells. Biochem Biophys Res Commun 1989;158:348-52.

8 Stark ME, Szurszewski JH. Role of nitric oxide in gastrointestinal and hepatic function and disease. Gastroenterology 1992;103:1928-49.

9 Szurszewski JH. A migrating electric complex of the canine small intestine. Am F Physiol 1969;217:1757-63.

10 Sarna SK. Cyclic motor activity; migrating motor complex. Gastroenterology 1985;89:894-913.

11 Sarr MG, Duenes JA, Tanaka M. A model of jejunoileal in vivo neural isolation of the entire jejunoileum: transplantation and the effects on intestinal motility. F Surg Res 1989;47:266-72. 